\title{
CORRECTIONS
}

\section{Publisher Correction: Bach's symphony for metastasis}

\section{Anna Dart}

Nature Reviews Cancer (2019) https://doi.org/10.1038/s41568-019-0182-0 Published online 22 July 2019

In the originally published Research Highlight, the ubiquitin ligase targeting BACH1 for degradation was incorrectly stated as CBL1-FBXO22. It should have read CRL1-FBXO22. This has now been corrected in all versions of the original Research Highlight.

https://doi.org/10.1038/s41568-019-0192-y I Published online 9 August 2019 